\title{
Elecciones presidenciales en Malawi 2014: ¿un cambio en el poder o más de lo mismo?
}

\author{
Malawi Presidential \\ Elections 2014: A Change \\ in Power or More of the Same?
}

\author{
ÓSCAR ALARCÓN JIMÉNEZ \\ Consejo de Europa
}

\section{Importancia de estas elecciones}

Tras la muerte repentina por infarto de Bingu wa Mutharika en abril de 2012, el cargo de presidente de Malawi pasó a manos de la vicepresidenta, Joyce Banda, que se convirtió en la primera mujer en Malawi, y segunda en África, en ostentar el cargo de jefe de Estado (presidenta). Banda fue la más sorprendida por esta encomienda, pues tras haber sido expulsada del partido del gobierno por llevar a cabo actividades contrarias al mismo, fundó en 2011 su propio partido político, el Partido Popular (PP), aunque conservó el cargo de vicepresidenta del país. Su nombramiento tuvo lugar a pesar de las maniobras de Peter Mutharika, hermano del difunto presidente, y de su partido político para impedir la sucesión legal. ${ }^{1}$

Si bien en un principio la inesperada sucesión de Banda fue recibida con buenos ojos por la ciudadanía y la comunidad internacional porque se pensó que pondría freno a los abusos cometidos durante años por Mutharika, la realidad fue muy distinta. Durante su gobierno, Malawi se vio inmerso en una gran inflación y los casos de corrupción la salpicaron de

${ }^{1}$ Commission of Inquiry, "Malawi Full Inquiry Report on Bingu wa Mutharika's Death”, Nyasa Times, 7 de marzo de 2013. 
tal manera que su popularidad cayó por los suelos ${ }^{2}$ y puso en peligro la relación de confianza del país con los organismos internacionales y los países donantes.

Las elecciones del 20 de mayo de 2014 se presentaban como clave para el futuro del país. Por un lado, y por primera vez en su historia desde su independencia del Reino Unido en 1964, Malawi ${ }^{3}$ celebraba en una misma jornada tres importantes comicios: presidenciales, parlamentarios y locales (elecciones tripartitas). Por otro lado, Joyce Banda, Peter Mutharika y otros 10 candidatos presidenciales se enfrentaban en una contienda electoral sin precedentes para obtener democráticamente el cargo de presidente de Malawi. Reto difícil en un país donde la corrupción ${ }^{4}$ y los métodos dictatoriales conservan, todavía hoy, raíces profundas en todos sus niveles. Finalmente, Joyce Banda no sólo buscaba en las urnas la legitimidad necesaria para ser nombrada presidenta electa, desde que asumiese el cargo en 2012, sino que además veía un refrendo a su labor presidencial durante los dos últimos años.

\section{Elecciones en juego}

Más de 7.5 millones de malauíes (46\% hombres, 54\% mujeres) estaban convocados a una jornada electoral única en la que se celebraban simultáneamente tres importantes comicios:

- Elecciones presidenciales: 12 candidatos $^{5}$ aspiraban a obtener el cargo de presidente del país durante un mandato de

${ }^{2}$ Nyasa Times, "Cashgate: Major dent on JB’s Credibility”, 15 de febrero de 2014.

${ }^{3}$ Para mayor información sobre el proceso de transición política de Malawi y las características de las diferentes elecciones democráticas, consúltese Hilda Varela, "Las elecciones generales de 2004: Sudáfrica y Malawi", Estudios de Asia y África, vol. 39, núm. 2 (124), mayo-agosto de 2004, pp. 421-444.

${ }^{4}$ Malawi ocupa el puesto 91 de 177 países en la estadística que publica anualmente Transparencia Internacional para medir los niveles de corrupción en el sector público de los distintos países. Transparency International, Corruption Perceptions Index 2013.

${ }^{5}$ Joyce Banda (People's Party), Arthur Peter Mutharika (Democratic Progressive Party), Atupele Muluzi (United Democratic Front); Lazarus Chakwera (Malawi Congress Party), George Mnesa (Malawi Forum for Unity and Development), Aaron 
cinco años. Sin embargo, sólo cuatro de ellos tenían grandes posibilidades de ganar: Joyce Banda (PP), Peter Mutharika (Partido Democrático Progresista, DPP por sus siglas en inglés), el reverendo Lazarus Chakwera (Partido del Congreso de Malawi, MCP por sus siglas en inglés) y Atupele Muluzi (Frente Democrático Unido, UDF por sus siglas en inglés), hijo del antiguo presidente Bakili Muluzi. A la luz de los resultados, Peter Mutharika ganó estos comicios con $36.40 \%$ de los votos.

- Elecciones parlamentarias: 17 fueron los partidos políticos que presentaban candidaturas para cubrir, durante un mandato de cinco años, los 193 escaños del Parlamento. En términos de resultados, el Parlamento siguió una tendencia similar al color político de las elecciones presidenciales.

- Elecciones locales: no se celebraban desde el año 2000, lo que implicaba una clara violación de la norma fundamental. Por aquel entonces, $y$ debido a restricciones presupuestarias, el presidente Bingu wa Mutharika no dudó en anular las elecciones locales de 2005. Cesó en pleno a la Comisión Electoral de Malawi (MEC, por sus siglas en inglés) por su presunta malversación de caudales públicos, censura que impidió la celebración de los comicios locales de 2010, y reincorporó al personal de la MEC en abril de 2011. Tras su muerte repentina, la nueva presidenta Joyce Banda nombró a nuevos comisarios.

\section{Análisis electoral}

La lectura en clave política de estas elecciones es que pueden entenderse como un paso adelante en el proceso de democratización de Malawi. Si bien la elección en las urnas de un nuevo gobierno es un indicador de la madurez democrática de un país, cabe destacar que la forma en la que se llevó a cabo

Davis Chester Katsonga (Chipani Cha Pfuko), Mark Katsonga (hermano de Aaron Davis Chester Katsonga) (People's Progressive Movement), Kamuzu Walter Chibambo (People's Transformation Party), James Mbowe Nyondo (National Salvation Front), Pastora Helen Singh (United Independence Party), Friday Anderson (New Labour Party) y John Chisi (Umodzi Party). 
puso en duda el propio proceso al que se enfrentó el país. Serios problemas logísticos empañaron estas elecciones desde el inicio. Por otro lado, aunque los altercados tras las elecciones no sumieron al país en la violencia, ${ }^{6}$ fueron muchas las voces críticas que impugnaron tanto los resultados, calificándolos de fraudulentos, ${ }^{7}$ como el propio proceso, cuestionado en términos de integridad y credibilidad tras las numerosas evidencias de anomalías ${ }^{8}$ detectadas, todas ellas reconocidas por la Comisión Electoral de Malawi.

\section{Principales dificultades electorales}

Unas listas electorales no del todo exactas, dificultades logísticas y una cuestionable gestión de los resultados ${ }^{9}$ fueron, entre otros, los principales problemas registrados.

La MEC, órgano constitucional creado en virtud del artículo 75 de la Constitución de Malawi para supervisar y dirigir la conducta en las elecciones, participó en las fases preparatorias de configuración de las listas electorales a través del Foro Consultivo Nacional de Elecciones (National Elections Consultative Forum).${ }^{10}$ Sin embargo, a pesar de que las listas electorales contenían alguno que otro contratiempo, esto no puso en peligro la integridad de los comicios.

Las dificultades logísticas fueron otro de los rompecabezas que empañaron indudablemente la jornada, principalmente por la escasez e impresión tardía del material electoral, esencial-

${ }^{6}$ Malawi Voice, "Blantyre on Fire", 20 de mayo de 2014.

7 AFP, "Malawi Election marred Riots Allegations Foul Play", The Guardian, 21 de mayo de 2014.

${ }^{8}$ Diana Cammack, "Why Malawi took so Long to declare an Election Winner", The Guardian, 30 de mayo de 2014.

${ }^{9}$ European Union Election Observation Mission-Malawi 2014, Preliminary Statement. Malawians succeeded to vote despite Considerable Organisational Shortcomings, Blantyre, 22 de mayo de 2014.

${ }^{10}$ El Foro Consultivo Nacional de Elecciones (NECOF por sus siglas en inglés) se instituyó en 2004 para promover la coexistencia pacífica y hacer frente a las diferencias que pudiera haber entre la Comisión Electoral de Malawi (MEC) y sus interlocutores en la gestión del proceso electoral. Su objetivo no era otro que el de mejorar la transparencia y la responsabilidad de la MEC ante la población. Este Foro está compuesto, entre otros, por líderes de partidos políticos, organizaciones de la sociedad civil, organizaciones no gubernamentales, medios de comunicación, etcétera. 
mente las papeletas, una apertura con retraso de los colegios electorales y la realización del escrutinio más allá de las elecciones, es decir, durante tres días consecutivos. ${ }^{11}$ Estas complicaciones impidieron a muchos ejercer su derecho de voto durante los horarios establecidos (de seis de la mañana a seis de la tarde).

La gestión de los resultados electorales fue un tanto caótica ${ }^{12}$ en la medida en que algunas hojas electorales no contenían las firmas necesarias y obligatorias (ni de los presidentes del Colegio electoral ni de los responsables políticos presentes) para declarar la validez del voto; ciertos resultados habían sido alterados o eran ilegibles; se confirmaron discrepancias numéricas en los colegios electorales entre los resultados obtenidos por cada uno de los candidatos y el número total de votos emitidos; se apreciaron errores entre el número de votos emitidos por cada mesa electoral y el número total de votos contabilizados por el Colegio electoral, ${ }^{13}$ hecho que favorecía a ciertos candidatos, etcétera.

Así pues, la MEC contabilizó más de 450 quejas sobre la integridad de los resultados. ${ }^{14}$

\section{¿Es la MEC la causa de todos los males?}

Las autoridades encargadas de las elecciones manifestaron que la falta de presupuesto de la MEC y el papel ineficaz de los donantes contribuyó a este cúmulo de circunstancias, esto es, a la falta de materiales electorales y a la impresión tardía de las papeletas electorales. ${ }^{15}$

Sin embargo, baste recordar que la autoridad organizadora, en este caso la MEC, es la principal responsable de un proce-

${ }^{11}$ Afp, "Malawi Election marred Riots Allegations Foul Play", op. cit.

12 Baldwin Chiyamwaka, "Malawi 2014 Tripartite Elections: Electoral Governance Tested", presentación en el Seminario sobre Elecciones en Malawi, Embajada del Reino de los Países Bajos en Pretoria, 25 de junio de 2014.

${ }^{13}$ European Union Election Observation Mission, Final Report Malawi 2014: Tripartite Elections, Presidential, Parliamentary and Local Council, 2014, Bruselas, European External Actions Service, 2014.

${ }^{14}$ Rex Chikoko, "Donors exposed", The Nation on Sunday, 8 de junio de 2014.

${ }^{15}$ Idem. 
dimiento eficaz y eficiente. En estos comicios se produjeron envíos de las papeletas a lugares equivocados, se distribuyeron tardíamente materiales sensibles y hubo retraso en la apertura de los colegios electorales. ${ }^{16}$ A título de ejemplo, nadie podía haberse imaginado que tan sólo a dos calles de la sede central de la MEC los colegios electorales no dispondrían de papeletas el mismo día de las elecciones, lo que les impidió abrir el Colegio electoral.

Tanto el Programa de Desarrollo de las Naciones Unidas (PNUD), que tenía un proyecto conjunto ${ }^{17}$ con el gobierno de Malawi ${ }^{18}$ y gestionaba el fondo de donantes electorales, como la MEC, admitieron que los socios donantes habían dejado de aportar casi la mitad de su presupuesto para apoyar en estos comicios. ${ }^{19}$ Así pues, la falta de fondos provocó que la mayoría del personal electoral recibiera formación apenas unos días previos a las elecciones, lo que impidió que desempeñara correctamente su trabajo y, en consecuencia, cometieron errores a la hora de procesar los datos.

Estos elementos provocaron tal malestar en la población que hubo ciertos altercados en la ciudad de Blantyre durante la jornada electoral. ${ }^{20}$

\section{La figura de Joyce Banda}

Durante la campaña electoral, Joyce Banda estuvo ausente de la escena nacional; no participó en ninguno de los debates pre-

${ }^{16}$ Nyasa Times Reporter, "Malawi Voting opens with Numerous Complaints", Nyasa Times, 20 de mayo de 2014.

${ }^{17}$ El gobierno de Malawi, a través del Ministerio de Hacienda, la MEC y el PNUD, dispone de un proyecto de tres años para apoyar las elecciones y el ciclo electoral hasta 2016, financiado por el Departamento Británico para el Desarrollo Internacional, la Unión Europea, Irlanda, Noruega, Japón y el PNUD. Este proyecto constituye alrededor de $40 \%$ del presupuesto de las elecciones de 2014 y cubre los gastos de asistencia técnica y la adquisición de materiales electorales seleccionados (United Nations Development Programme, "Malawi Electoral Cycle Support", s.f.).

${ }_{18}$ Malawi Electoral Commission, Report on Tripartite Elections May 2014, Blantyre, Malawi, 2014.

${ }^{19}$ Chikoko, "Donors exposed", op. cit.

${ }^{20}$ AFP, "Malawi Election marred Riots Allegations Foul Play", op. cit. 
sidenciales alegando estar muy ocupada. ${ }^{21}$ Sin embargo, destacaron sus apariciones públicas fuera del país, tanto en organizaciones internacionales como en diferentes países africanos.

A pesar de reducir su propio sueldo, prometer reformas importantes, reactivar la ayuda de los donantes occidentales y poner a la venta el jet de su predecesor, Joyce Banda no consiguió ganar la simpatía de los malauíes debido, entre otras cosas, a las medidas de austeridad que adoptó para reparar la economía nacional, como la devaluación de $49 \%$ de la moneda local ${ }^{22}$ (el $k$ wacha) y el aumento de los precios del petróleo en tres ocasiones, que había sido exigido por organismos internacionales y por otros donantes occidentales. Los efectos colaterales de tales medidas no se hicieron esperar: los precios de los productos básicos se encarecieron más de 50\%, y la inflación aumentó, de tal forma que la mayoría de las familias no podían cubrir sus necesidades alimentarias básicas.

Los casos de corrupción, principalmente el escándalo Cashgate, ${ }^{23}$ destapados durante su mandato hicieron que su popularidad cayera por los suelos.

Cuando la presidenta Banda pretendió invalidar las elecciones y anunció una nueva convocatoria en un plazo de 90 días, antes incluso de anunciar los resultados de los comicios, alteró el clima electoral. Citando la sección $88^{24}$ de la Consti-

${ }^{21}$ Patrick Mwanza, "Boycotting Debates: Is God Secret Weapon for Joyce Banda?", Nyasa Times, 22 de abril de 2014.

${ }^{22}$ Godfrey Mapondera y David Smith, "Malawian President Joyce Banda faces Electoral Humiliation - and Possibly Jail", The Guardian, 29 de mayo de 2014.

${ }^{23}$ Caso Cashgate: nombre dado por la prensa a un importante escándalo de corupción ocurrido en Malawi en el que se vieron implicados muchos altos cargos políticos y funcionarios malauíes incapaces de demostrar el origen de grandes cantidades de dinero, objeto de apropiación indebida. Para lavar su imagen pública, la presidenta Joyce Banda destituyó al gobierno en pleno el 10 de octubre de 2013 y, cuatro días más tarde, nombró otro sin contar con los antiguos ministros de Justicia y Finanzas, hecho que aumentó aún más las especulaciones sobre la implicación de ambos en dicho escándalo. Asimismo, solicitó a una consultora del Reino Unido la realización de un informe independiente que diera luz a lo sucedido. Dicho informe (National Audit Office Malawi, Report on Fraud and Mismanagement of Malawi Government Finances, Londres, Baker Tilly, 21 de febrero de 2014) no hizo más que exponer la magnitud del caso y las irregularidades (pagos a empresas por un valor de 14.5 millones dólares por servicios no suministrados, pagos sin documentos justificativos, etcétera). El informe concluye que se defraudó al Estado malauí por unos 32 millones de dólares (casi 1\% del PIB anual de Malawi) en sólo seis meses.

${ }^{24}$ Sección 88 (2): "El presidente deberá proporcionar liderazgo ejecutivo en 
tución de Malawi, Banda justificó la nulidad de las elecciones alegando irregularidades en el conteo, lo que a su modo de ver comprometía la integridad de los sufragios. ${ }^{25}$ Argumentó igualmente que, según la Constitución, era su deber proteger la República de Malawi y por eso anulaba el proceso electoral y posponía las elecciones 90 días. Sin embargo, afirmó que no se presentaría como candidata.

Así pues, Banda justificó esta actuación aduciendo que daba a los malauíes la oportunidad de escoger de manera "justa y democrática" un nuevo líder. El anuncio lo hizo cuando los resultados preliminares del conteo ya reflejaban una derrota en toda regla de su partido frente a sus adversarios políticos $(23 \%$ a favor de Banda frente a $42 \%$ de Peter Mutharika).

Ante este inesperado aviso, muchos ciudadanos salieron a la calle para manifestar su descontento; paralelamente, los líderes políticos acudieron a la justicia. Aunque el propósito de la presidenta de anular las elecciones fue revocado por el Tribunal Supremo, ${ }^{26}$ la MEC reconoció que se habían detectado anomalías que ponían en tela de juicio la integridad de todo el proceso. $^{27}$

Sorprende que Banda haya hecho este anuncio, ya que únicamente al Tribunal Supremo, y no al jefe del Estado, le compete legalmente anular las elecciones. Varias son las hipótesis de calado político que explicarían sus motivos:

- Si bien Banda no ganaba nada con el anuncio, la realidad es que estaba perdiendo en las urnas la batalla contra Peter Mutharika, quien ya había intentado eliminarla de la escena política, concretamente tras la muerte de su hermano Bingu al impedir su nombramiento como presidenta. Esto, sin duda, afectaba su orgullo.

- Al anular las elecciones, Banda podría pretender negociar con uno de los candidatos en liza, y futuro presidente, su

interés de la unidad nacional, de conformidad con esta Constitución y las leyes de la República".

${ }_{25}$ Simon Allison, "Dispute over Malawi Election Results ends Joyce Banda's Political Sainthood", The Guardian, 27 de mayo de 2014.

${ }^{26}$ Mabvuto Banda y Frank Phiri, "Malawi High Court Rejects President's Call to Annul Elections", Reuters, 24 de mayo de 2014.

${ }^{27}$ Allison, "Dispute over Malawi Election Results...”, op. cit. 
situación política tras su salida del gobierno, ya que según algunas especulaciones sería acusada de corrupción en caso de que Mutharika llegase al poder. ${ }^{28}$

\section{Laberinto judicial y resultados electorales}

Una semana después de la jornada electoral y antes de anunciar los resultados finales, la MEC notificó, mediante orden judicial, su intención de realizar un recuento de votos debido a importantes irregularidades detectadas. Para hacerlo, se pretendía ampliar el mandato constitucional de ocho a treinta días y acallar las voces críticas que tachaban las elecciones de fraudulentas. Sin embargo, la iniciativa no fue bien recibida por el DPP, cuyo candidato, Peter Mutharika, estaba en esos momentos a la cabeza de los resultados. Además, el 30 de mayo los partidarios del recuento convocaron a una marcha en la ciudad de Mangochi, en la que hubo graves enfrentamientos con la policía y murió uno de los manifestantes. ${ }^{29}$

En un clima de acusaciones cruzadas, y en el último día del plazo legal para anunciar los resultados electorales - poco antes de media noche-, el juez del Tribunal Supremo encargado del caso $^{30}$ declaró que aun cuando la MEC tenía competencia para ejecutar las medidas necesarias para proporcionar las cifras finales (incluido el recuento de votos), el plazo de ocho días no podía ser ampliado por una decisión judicial. Esto provocó que la MEC se apresurara a anunciar los resultados presidenciales apenas una hora después de hacerse pública la sentencia, debido a la expiración del plazo legal.

${ }^{28}$ SAPA-AFP, "Mutharika wins Malawi's Disputed Presidential Poll”, Mail E Guardian, 31 de mayo de 2014.

${ }^{29}$ BBC News, "Malawi protester killed in poll recount clash", 30 de mayo de 2014.

${ }^{30}$ Este caso había sido asignado originariamente al juez del Tribunal Supremo D. Healy Potani, quien tuvo que retirarse por un conflicto de intereses, ya que su hermano era miembro de la MEC. Así es como este caso llegó a manos del juez D. Kenyatta Nyirenda. 


\section{Análisis del fallo judicial}

En el marco de unas elecciones polémicas, el juez Kenyatta Nyirenda tuvo la ardua tarea de dilucidar tres cuestiones extremadamente sensibles:

- Si la MEc había incurrido en desacato debido a requerimientos presentados durante el conteo de los votos.

- Si la ley malauí otorgaba a la MEc la posibilidad de llevar a cabo un recuento de votos.

- Si el mandato constitucional de ocho días otorgado a la MEC para facilitar los resultados podía extenderse para realizar un recuento.

Tras un análisis pormenorizado de los hechos, el juez dio respuesta a estas tres incógnitas en una misma sentencia argumentando que:

- Respecto a la primera cuestión, los demandantes no habían proporcionado la información necesaria para proceder a audiencias por desacato.

- Respecto a la segunda cuestión, el conteo y el recuento de votos se circunscribían a las competencias otorgadas a la MEC por la ley y la Constitución.

- Respecto a la tercera cuestión, el juez hizo una interpretación stricto sensu de la ley al afirmar que sólo debía interpretarla y no crearla, ${ }^{31}$ con lo que denegó la extensión del mandato constitucional.

El razonamiento jurídico del juez Nyirenda muestra su compromiso por el respeto al Estado de derecho y el cumplimiento del imperio de la ley.

${ }^{31}$ Zawadi Chilunga-Nyasa Times, "Court Rules MEC should declare Winner of Malawi Presidential Polls”, Nyasa Times, 30 de mayo de 2014. 


\section{Resultados electorales ${ }^{32}$}

\section{Elecciones presidenciales}

Con $36.40 \%$ de los votos, Peter Mutharika ganó estos comicios, seguido de cerca por Lazarus Chakwera con $27.80 \%$ de los votos. Joyce Banda quedó en tercer lugar con $20.20 \%$ y Atupele Muluzi obtuvo $13.70 \%$. De los casi 7.5 millones de electores malauíes, ${ }^{33} 70 \%$ (5285278) se acercó a las urnas a manifestar su voto. Por regiones, cabe destacar un claro vencedor en cada una de ellas:

- A pesar de haber sido un feudo del antiguo presidente, Joyce Banda arrasó en la región del norte con $56.27 \%$ de los votos, seguida por Peter Mutharika (21.84\%).

- El reverendo Lazarus Chakwera ganó en la región central con $58.33 \%$, seguido por Peter Mutharika (20.17\%).

- El profesor Peter Mutharika (56.71\%) arrasó en la región del sur, más poblada, seguido por Atupele Muluzi (23.63\%).

Elecciones parlamentarias

El siguiente cuadro muestra los resultados del arco parlamentario malauí:

\begin{tabular}{|ccccccccc|}
\hline Partido político & Independientes & DPP & MCP & PP & UDF & AFORD & CCP & Total \\
\hline Escaños & 52 & 50 & 48 & 26 & 14 & 1 & 1 & 192 \\
\hline
\end{tabular}

Es necesario explicar los siguientes elementos importantes:

- El gran número de candidatos independientes se explica por el procedimiento interno de nominación de candidatos,

${ }^{32}$ Página web oficial de la Malawi Electoral Commission: www.mec.org.mw.

${ }^{33}$ Es importante señalar que, a pesar de que las distinciones étnicas tuvieron mucha importancia durante el siglo pasado, hoy en día han disminuido hasta tal punto que no hay fricción interétnica significativa. Los principales grupos étnicos de Malawi son los tumbuka y los tonga en la región del norte, los chewa constituyen cerca de $90 \%$ de la población de la región central, y los nyanja y los yao, estos últimos principalmente musulmanes, predominan en la región sur. 
no exento de problemas, que lleva a un gran número de aspirantes descontentos a presentarse como independientes.

- El número total de escaños no llega a los 193 porque las elecciones parlamentarias en la circunscripción de Blantyre Norte fueron diferidas tras el fallecimiento por muerte natural de un candidato parlamentario.

- A la luz de los resultados, como ningún partido político goza de mayoría absoluta en el Parlamento, los acuerdos políticos serán más que necesarios para sacar adelante cualquier iniciativa.

- A pesar de los esfuerzos para aumentar el número de parlamentarias, sólo 30 mujeres obtuvieron escaños. La representación femenina en el Parlamento de Malawi pasó de 22 a $16 \%$, cifra muy inferior al objetivo de $50 \%$ establecido en el Protocolo de Género y Desarrollo ${ }^{34}$ de la Comunidad de Desarrollo de África Austral (SADC por sus siglas en inglés), del que Malawi es firmante.

\section{Elecciones locales}

El siguiente cuadro muestra los resultados de las elecciones municipales por partidos políticos:

\begin{tabular}{|c|c|c|c|c|c|c|c|c|c|}
\hline Partido político & DPP & MCP & PP & UDF & Independiente & ССР & AFORD & NASAF & Total \\
\hline Municipios & 165 & 131 & 65 & 57 & 35 & 2 & 1 & 1 & 457 \\
\hline
\end{tabular}

El número total de municipios es de 462. Sin embargo, en cinco municipalidades (Zgeba, Lifupa, Kandeu, Lisonjala y Mbalachnada) se aplazaron las elecciones debido al fallecimiento por muerte natural de los candidatos. En materia de género, 56 municipalidades estarán gobernadas por mujeres y 401 por hombres.

Los resultados de las elecciones municipales reflejan la tendencia política de los resultados de las elecciones presidenciales y parlamentarias.

${ }^{34}$ Southern African Development Community, SADC Protocol on Gender and Development, 17 de agosto de 2008, Art. 12.1. 


\section{La administración electoral: la Comisión Central Electoral (MEC)}

La percepción negativa sobre la actuación de la MEC en estas elecciones ha sido un elemento crucial para la credibilidad de estos comicios. En un principio, la MEC había encarado su gestión y su trabajo de manera positiva, e incluso fue aplaudida por su proceso de consulta y transparencia. Aunque los nombramientos de los comisarios fueron objeto de fuertes críticas en el pasado, la situación para estos comicios fue distinta gracias al nombramiento como presidente de la MEC del juez Mbendera, que contaba con el beneplácito de gran parte de la población. ${ }^{35}$ Baste recordar que la MEC está compuesta por un presidente, juez nombrado por el consejo del Poder Judicial (órgano de regulación de la Judicatura de Malawi), y seis comisarios (como mínimo), nombrados por el presidente de Malawi tras consulta con los líderes de los partidos políticos representados en el Parlamento, ${ }^{36}$ por un periodo de cuatro años y con la posibilidad de ser reelegidos para un nuevo mandato.

La MEC fue muy criticada en estos comicios por su incapacidad para:

- Tratar las quejas: en lugar de resolver las dificultades planteadas por las partes interesadas, se dedicó a distender tales asuntos sin dar una solución concreta al problema, lo que llevó a la acumulación de reclamos sin respuesta, tal como se apreció el día de las elecciones y durante el conteo.

- Seguir un calendario de actividades: el calendario del registro electoral no cesó de cambiar aleatoriamente; ${ }^{37}$ tampoco dispuso de una fecha fija en la que se fuera a efectuar el transporte de materiales a los colegios electorales de todo el país. ${ }^{38}$

${ }^{35}$ Face of Malawi, "Joyce Banda appoints Mbendera as MEC chair, Msosa out", 13 de octubre de 2015. 1998.

${ }^{36}$ Sección 4 del Acta de la Comisión Electoral (ECA por sus siglas en inglés) de

37 The Nation, “MEC broke, Elections under Threat”, 17 de mayo de 2013.

${ }^{38}$ Brenda Nkosi, "2014 Election Challenges Rooted from Inadequate Transportation”, Malawi Neres Agency Online, 1 de septiembre de 2014. 
- Atajar rumores de fraude: como el fraude ha sido parte de las pasadas ${ }^{39} \mathrm{y}$ de las actuales ${ }^{40}$ elecciones, se cree que la MEC, al ser nombrada por el gobierno, es incapaz de hacerlas creíbles. ${ }^{41}$ Lamentablemente, estos rumores no se cortaron mediante investigaciones exhaustivas y aportando pruebas fehacientes de que nunca existieron. Más bien se trataron como meras invenciones.

- Desarrollar una política logística: el retraso en la apertura de los colegios electorales respecto a la hora legalmente fijada y la imposibilidad de entregar el material adecuado provocó fuertes protestas en muchos colegios electorales y condujo a que la votación se extendiera durante tres días. $^{42}$

Banda también contribuyó a sembrar dudas sobre la fiabilidad del trabajo desempeñado por la MEC al acusar a un partido político, sin decir cuál, de infiltrarse y hackear el sistema informático que transmitía los resultados electorales a la MEC. ${ }^{43}$ Esta afirmación obligó al presidente de la Comisión a desmentir tales declaraciones pero a reconocer, sin embargo, fallos en el siste$\mathrm{ma}$, hecho que la obligó a realizar un conteo manual de votos.

En su defensa cabe decir que, por un lado, el gobierno de Malawi nunca antes había organizado unas elecciones de tal magnitud (tripartitas) y, por otro, que la MEC infravaloró los desafíos que conllevaba hacerlo. A la luz de lo sucedido, surge la necesidad de revisar el marco electoral de Malawi, concretamente la legislación, que ha sido deficiente para ofrecer soluciones a lo que sucedió en el país, sobre todo en lo que se refiere a las

39 BBC Neres, "Malawi President Wins Re-election", 22 de mayo de 2009; Nyasa Times, "DPp fears Electoral Fraud, gives Malawi Electoral Body 48 hours Ultimum on Civic Education", 11 de julio de 2013.

${ }^{40}$ Reuters, "Citing Fraud, Malawi Leader Halts Vote and Stirs Protest", The New York Times, 24 de mayo de 2014; Neres24, "Malawi to Recount Votes after Fraud Claims”, 25 de mayo de 2014.

${ }^{41}$ Traci D. Cook y Chris Chisoni, Preparing for elections: Citizens Share Their Vieres on Key Issues \& the 2014 Vote, s.l., Catholic Commission for Justice and PeaceNational Democratic Institute for International Affairs, 7 de mayo de 2014, p. 73.

${ }^{42}$ Star Africa, "Malawi pols Extended to Third Day", 22 de mayo de 2014.

${ }^{43}$ BBC New, "Malawi Poll Marred by Rigging, says President Joyce Banda", 22 de mayo de 2014. 
disputas, quejas e irregularidades surgidas durante el proceso electoral. El espectáculo judicial ${ }^{44}$ en el que se vio inmerso el país puso en tela de juicio la capacidad de la MEC para gestionar correctamente los comicios.

\section{Perspectiva de futuro: los principales retos del nuevo presidente}

A la luz de los resultados electorales, varios aspectos marcan la impronta del país.

- Corrupción: en este clima de intrigas políticas y declaraciones de cantantes internacionales (Madonna no dudó en irrumpir durante la campaña electoral acusando a Joyce Banda de corrupción), ${ }^{45}$ la lucha contra la corrupción es uno de los desafíos a los que debe enfrentarse cuanto antes el nuevo presidente. En uno de los países más pobres del mundo, las consecuencias del mal gobierno y la corrupción son irreversibles. Es importante que Malawi adopte y cree, para su beneficio, normativas e instituciones debidamente aplicadas para erradicar esta lacra. Poco importa cuán polémico o incómodo sea. Peter Mutharika no puede mirar hacia otro lado cuando este problema está gangrenando al país. Debería explorar iniciativas que revelen las causas del conflicto y aporten soluciones. Los casos recientes de corrupción han traído como consecuencia que las ayudas monetarias a Malawi hayan cesado. ${ }^{46}$

${ }^{44}$ Baste recordar que la MEC tuvo que acudir en dos ocasiones al Tribunal Supremo: una vez para contrarrestar la decisión de la entonces presidenta Banda que quería anular las elecciones; y una segunda ocasión para solicitar una extensión del plazo legal del cómputo y realizar un conteo.

${ }^{45}$ Khanyo Olwethu Mjamba, "Madonna makes Statement on Malawi President", This is Africa, 27 de mayo de 2014.

${ }^{46}$ El Fondo Mundial de Lucha contra el Sida ha dejado de financiar ayuda para Malawi por un nuevo caso de corrupción. Presumiblemente, la Comisión Nacional contra el Sida (NAC por sus siglas en inglés) dio el dinero destinado a la campaña contra el sida a algunos periodistas y a otras iniciativas relacionadas con la esposa del jefe de Estado, con su etnia de origen e incluso con los servicios secretos. Wanga, Gwede-Nyasa Times, "Global Fund suspend Malawi Aids Funding: Crisis Looms over Nacgate", Nyasa Times, 5 de enero de 2015. 
- Electorado polarizado: durante su mandato, Peter Mutharika tendrá la ardua tarea de unir al país y reconciliarse con la región del norte de Malawi. Hasta entonces no conseguirá la estabilidad económica y política necesaria para el desarrollo del país.

Por un lado, debe convencer a los más de tres millones de votantes de que su manera de gobernar será muy diferente y más democrática que la de su hermano. A pesar de ser el vencedor de estas elecciones, Peter Mutharika obtuvo un porcentaje de votos (36\%) muy por debajo del logrado por su hermano (67\%). Con los actuales desafíos por los que atraviesa Malawi, será difícil construir, con dicho porcentaje, una situación democrática inclusiva en el país.

La reconciliación con la región del norte de Malawi no será fácil, pues ha pasado de $100 \%$ de votos obtenidos en los comicios de 2009 por su hermano Bingu wa Mutharika a apenas $22 \%$ en 2014. Cabe recordar que el gobierno del DPP, en el que Peter Mutharika fue ministro de Educación, instauró en las universidades de Malawi el sistema trimestral de selección, procedimiento de acceso que establece una cuota de plazas universitarias para estudiantes por región del país, independientemente de su resultado académico. Este sistema, muy criticado por los ciudadanos del norte, se opone al de méritos utilizado con anterioridad y que beneficiaba a aquellos estudiantes con mejores calificaciones, principalmente a los de la región del norte. Así pues, Peter Mutharika debe encontrar la manera de ganarse la confianza de una de las regiones más pobladas de Malawi.

- Establecer relaciones con países occidentales: la relación de Malawi con los países occidentales, principalmente donantes, se fue apagando a partir de 2012, lo que provocó que le retirasen su ayuda. El antiguo DPP ha dirigido su mirada más bien a Asia. Tanto es así que en su discurso inaugural del juramento de su nuevo cargo presidencial, Peter Mutharika manifestó que los países occidentales no eran los únicos aliados de Malawi y que el país se esforzaría por conseguir "nuevos amigos" en economías emergentes como Brasil, Ru- 
sia, India, China y Sudáfrica (BRICS)..$^{47}$ Desde la zona AsiaPacífico, y como guiño a estas declaraciones, la rápida respuesta del gobierno chino no se hizo esperar, y a través del portavoz de su Ministerio de Exteriores felicitó públicamente al nuevo presidente electo. ${ }^{48}$

- Las aguas de la discordia: en un intento de ganarse a la población, el actual presidente confirmó las reivindicaciones de su país sobre el lago Malawi, antiguamente llamado Nyasa, considerado como una zona rica en hidrocarburos (principalmente petróleo y gas), y hoy en día el ojo del huracán de un diferendo transfronterizo con su vecina Tanzania. Ambos países discuten el trazado de la frontera del lago y cada uno espera encontrar importantes reservas de hidrocarburos. Malawi reclama la totalidad de la superficie lacustre, incluidas las aguas cercanas a la costa de Tanzania (salvo aquella que se encuentre en Mozambique); y Tanzania demanda que la frontera internacional pase por la mitad del lago. La polémica está servida.

Ambos Estados se apoyan en un acuerdo de 1890 (Acuerdo anglo-alemán o Tratado de Heligoland-Zanzíbar), firmado entre el Reino Unido y Alemania, según el cual el gobierno colonial británico colocó las aguas del lago bajo la jurisdicción única del territorio de Nyasalandia. ${ }^{49}$ Las aguas del lago se comparten también con Mozambique, país que está, no obstante, fuera de la disputa.

Este diferendo lleva asociado consigo ciertos problemas de seguridad e inmigración para Malawi. La solución a la disputa es un desafío regional para la construcción de países prósperos que aspiran a vivir en paz y cimentar un bienestar común. Peter Mutharika deberá apostar por una solución satisfactoria si no quiere que la población malauí se sienta traicionada por su nuevo presidente.

${ }^{47}$ Bangkok Post, "Malawi's President seeks 'New Friends' in China, Russia”, 2 de junio de 2014.

${ }^{48}$ Xinhua News Agency, “China congratulates Malawi’s New President”, People’s Daily Online, 3 de junio de 2014.

${ }_{49}$ Protectorado británico establecido el 6 de julio de 1907. Desde 1964 se conoce al país con el nombre de Malawi. El 6 de julio de 1964 se proclamó la independencia del territorio y se convirtió en la República de Malawi. 
- Otros desafíos. El debate sobre la homosexualidad irrumpió en la campaña electoral debido a la presión de los colectivos LGBTI. Incluso Peter Mutharika negó las acusaciones vertidas en su contra por sus preferencias sexuales. Si bien Joyce Banda dejó en suspensión ${ }^{50}$ la ley que penaba la homosexualidad, ${ }^{51}$ el nuevo ministro de Justicia anunció en julio de 2014 que Malawi dejaría de arrestar a quienes mantuvieran relaciones sexuales con personas del mismo sexo y que se revisarían las leyes en contra de los homosexuales. ${ }^{52}$ El mensaje fue recibido como una batalla ganada por la comunidad LGBTI, que ha depositado muchas esperanzas en Mutharika.

La edad del nuevo presidente puede ser vista como una desventaja para su carrera profesional. A pesar de que Peter Mutharika contrajo matrimonio el 21 de junio de 2014 con su amiga y confidente de largo tiempo, Gertrude Maseko, sus 74 años pueden pasarle la factura durante esta legislatura.

\section{Conclusión}

Unas elecciones auténticas y periódicas son necesarias para legitimar un gobierno y reforzar la voluntad del pueblo, que es la base de la autoridad del poder público. ${ }^{53}$ Sin embargo, la celebración de elecciones per se no implica democracia; las elecciones son sólo un elemento más.

El papel que desempeña en unos comicios el organismo electoral es fundamental, ya que cualquier irregularidad durante el proceso, por mínima que sea, puede ser considerada, bien por la ciudadanía, bien por cualquiera de los grupos políticos en liza, como una artimaña fraudulenta para manipularlos en beneficio del partido en el poder. Este tipo de situaciones debería evitarse

${ }^{50}$ Godfrey Mapondera y David Smith, "Malawi suspends Anti-Gay Laws as MPs Debate Repeal", The Guardian, 5 de noviembre de 2012.

${ }^{51}$ Hasta 14 años pueden pasar en prisión los hombres y cinco años las mujeres (art. 153 y art. 137-A Código Penal).

52 Joseph Patrick McCormick, "Malawi to stop Gay People for having Gay Sex", Pink Neres, 15 de julio de 2014.

${ }^{53}$ Artículo 21.3 de la Declaración Universal de los Derechos Humanos. 
a toda costa, y si se cuenta con una planificación ordenada, tales problemas son fácilmente superables. De lo contrario, se pone en duda la credibilidad y la transparencia del proceso en sí. Desde un punto de vista objetivo, la MEC desarrolló, para estos comicios tripartitos, un plan estratégico detallado que contó con el beneplácito de los actores nacionales e internacionales. Sin embargo, las dificultades en la gestión de dicho plan antes, durante y después de los comicios sembraron serias dudas en la ciudadanía, tantas, que decidió salir a la calle a provocar incidentes, lo que contribuyó a aumentar la tensión. Una vez finalizados los comicios, y en aras de aprender de las lecciones pasadas, debería realizarse un informe objetivo que detallase las irregularidades que afectaron el proceso y su credibilidad, las posibles reformas legales necesarias y los retos logísticos, entre otros, a los que debe enfrentarse la MEC para mejorar futuras convocatorias electorales.

Un aspecto importante para evitar controversias es la norma de financiación de la campaña electoral. La falta en Malawi de una prohibición explícita del uso de los recursos del Estado para hacer campaña provoca gran confusión sobre los recursos utilizados por el partido gobernante y los recursos del Estado.

La posibilidad de que unos comicios desencadenen episodios de violencia pone de manifiesto la necesidad de contar con mecanismos sólidos y transparentes de gestión de los procesos electorales y de sus resultados. ${ }^{54}$ Tan importante como la efectividad del organismo electoral es su credibilidad. Así pues, si la ciudadanía y los partidos políticos tienen plena confianza en quienes dirigen el organismo electoral, habrá entonces mayores posibilidades de que se acepten los resultados. De lo contrario, el riesgo de inconformidad es alto.

Durante estas elecciones tripartitas destaca el papel de las fuerzas del orden, que son actores importantes en el marco de los comicios. En la mayoría de los países democráticos, los militares no tienen una función particular durante las elecciones. La protección de los colegios electorales no es responsa-

${ }^{54}$ International Peace Institute, Elections and Stability in West Africa: The Way Forward, Nueva York, International Peace Institute, 2012. 
bilidad de las fuerzas armadas, sino de la policía. Los soldados pueden tener un papel subsidiario en caso de necesidad. Esto es, en emergencias los militares pueden participar en la protección de la democracia, de las instituciones y del orden constitucional. En el caso de Malawi, y a pesar de los altercados durante los comicios, el ejército garantizó la estabilidad del país manteniendo la paz y complementando el papel de la policía a la hora de mantener el orden. ${ }^{55}$ Este deseo de paz (elecciones libres de toda violencia) es la petición que firmaron los candidatos políticos en la Declaración de Paz de Lilongüe ${ }^{56}$ del 10 de mayo de 2014.

De la misma manera, en un Estado de derecho, un Poder Judicial independiente es necesario para resolver de modo efectivo cualquier controversia durante las elecciones, y más si son controvertidas. En este caso concreto, destaca la actuación ejemplar y neutral del juez del Tribunal Supremo de Malawi, que, con su sentencia, respaldó el respeto al Estado de derecho y a la democracia.

Antes incluso de la sentencia del Tribunal Supremo, la mayoría de las misiones de observación electoral que participaron en las elecciones tripartitas de Malawi ${ }^{57}$ habían declarado que éstas habían sido libres, justas y creíbles, señalando que las irregularidades observadas no fueron lo suficientemente graves como para poner en peligro la integridad de los comicios. Otra cosa fue si la MEC, único árbitro de las elecciones, llegó a la misma conclusión.

$¿$ Deben entenderse estas elecciones como una historia de éxito de la democracia? Sólo el tiempo nos dará la respuesta.

${ }^{55}$ Green Muheya-Nyasa Times, "Polling Station set on Fire, MEC says Malawi Army to 'Expand its Presence", Nyasa Times, 20 de mayo de 2014; Ufulu, "Malawi Army and Police assure Malawians of a Secure and Safe Election", The Maravi Post, 20 de mayo de 2014. de 2014

${ }^{56}$ Public Affairs Committee, Lilongree Peace Declaration, Lilongüe, 10 de mayo

${ }^{57}$ Misiones de observación electoral que participaron en estas elecciones tripartitas: Unión Africana, Unión Europea, Naciones Unidas, Commonwealth, Malawi Council of Churches, Malawi Electoral Support Network (MESN), National Initiative for Civic Education (NICE Trust), Electoral Commissions Forum of the SADC Countries (ECF-SADC), SADC Parliamentary Forum Election Observation, SADC Electoral Observation Mission, Zimbabwe Elections Support Network (ZESN). 


\section{Bibliografía}

AfP, "Malawi Election marred Riots Allegations Foul Play", The Guardian, 21 de mayo de 2014.

Allison, Simon, "Dispute over Malawi Election Results ends Joyce Banda's Political Sainthood”, The Guardian, 27 de mayo de 2014.

Asamblea General de las Naciones Unidas, Declaración Universal de los Derechos Humanos, 10 de diciembre de 1948. [ < http://www. ohchr.org/EN/UDHR/Documents/UDHR Translations/spn. pdf $>$, consultado en enero de 2015.]

Banda, Mabvuto y Frank Phiri, "Malawi High Court Rejects President's Call to Annul Elections", Reuters, 24 de mayo de 2014.

Bangkok Post, "Malawi's President seeks 'New Friends' in China, Russia", 2 de junio de 2014.

$B B C$ New, "Malawi Poll Marred by Rigging, says President Joyce Banda", 22 de mayo de 2014.

$B B C$ Neres, "Malawi President wins Re-election", 22 de mayo de 2009.

$B B C$ Neres, "Malawi protester killed in poll recount clash", 30 de mayo de 2014.

Cammack, Diana, "Why Malawi took so Long to declare an Election Winner", The Guardian, 30 de mayo de 2014.

Cнткоко, Rex, "Donors exposed", The Nation on Sunday, 8 de junio de 2014.

Chilunga, Zawadi-Nyasa Times, "Court Rules MEC should declare Winner of Malawi Presidential Polls", Nyasa Times, 30 de mayo de 2014.

ChIYAmwaKa, Baldwin, "Malawi 2014 Tripartite Elections: Electoral Governance Tested", presentación en el Seminario sobre Elecciones en Malawi, Embajada del Reino de los Países Bajos en Pretoria, 25 de junio de 2014.

Commission of Inquiry, "Malawi Full Inquiry Report on Bingu wa Mutharika's Death", Nyasa Times, 7 de marzo de 2013.

Cook, Traci D. y Chris Chisoni, Preparing for elections: Citizens Share Their Vieres on Key Issues \& the 2014 Vote, s.1., Catholic Commission for Justice and Peace-National Democratic Institute for International Affairs, 7 de mayo de 2014.

European Union Election Observation Mission, Final Report Malawi 2014: Tripartite Elections, Presidential, Parliamentary and Local Council, 2014, Bruselas, European External Actions Service, 2014. [ < http://eeas.europa.eu/eueom/missions/2014/malawi/ pdf/eueom-malawi2014-final-report_en.pdf $>$, consultado en enero de 2015.] 
European Union Election Observation Mission-Malawi 2014, Preliminary Statement. Malawians succeeded to vote despite Considerable Organisational Shortcomings, Blantyre, 22 de mayo de 2014. [ < http://eeas.europa.eu/archives/eueom/ missions/2014/malawi/pdf/eueom-malawi2014-preliminarystatement_en.pdf $>$, consultado en enero de 2015.]

Face of Malawi, "Joyce Banda appoints Mbendera as MEC chair, Msosa out”, 13 de octubre de 2015.

Gwede, Wanga-Nyasa Times, "Global Fund suspend Malawi Aids Funding: Crisis Looms over Nacgate”, Nyasa Times, 5 de enero de 2015.

International Peace Institute, Elections and Stability in West Africa: The Way Forward, Nueva York, International Peace Institute, 2012. Malawi Electoral Commission, Report on Tripartite Elections May 2014, Blantyre, Malawi, 2014. [<http://www.mec.org.mw/ files $/ 2014 \% 20$ election $\% 20$ report $\% 20$ redone.pdf $>$, consultado en enero de 2015.]

Malawi Voice, "Blantyre on Fire", 20 de mayo de 2014.

Mapondera, Godfrey y David Smith, "Malawi suspends Anti-Gay Laws as mps Debate Repeal", The Guardian, 5 de noviembre de 2012.

Mapondera, Godfrey y David Smith, "Malawian President Joyce Banda faces Electoral Humiliation - and Possibly Jail", The Guardian, 29 de mayo de 2014.

McCormick, Joseph Patrick, "Malawi to stop Gay People for having Gay Sex”, Pink Neres, 15 de julio de 2014.

Muheya, Green-Nyasa Times, "Polling Station set on Fire, MEC says Malawi Army to 'Expand its Presence", Nyasa Times, 20 de mayo de 2014.

MwanZa, Patrick, "Boycotting Debates: Is God Secret Weapon for Joyce Banda?”, Nyasa Times, 22 de abril de 2014.

National Audit Office Malawi, Report on Fraud and Mismanagement of Malawi Government Finances, Londres, Baker Tilly, 21 de febrero de 2014. [ < https://www.gov.uk/government/uploads/ system/uploads/attachment_data/file/285877/20140221_National_Audit_Office_Malawi_-_Forensic_Audit_Report_-_FINAL $\overline{I S S U E}_{\bar{D}} \cdot \mathrm{pdf}>\overline{ }$, , consultado en enero de 2015.]

Nerws24, "Malawi to Recount Votes after Fraud Claims", 25 de mayo de 2014.

Nkosi, Brenda, "2014 Election Challenges Rooted from Inadequate Transportation", Malawi News Agency Online, 1 de septiembre de 2014. 
Nyasa Times, “Cashgate: Major dent on JB’s Credibility”, 15 de febrero de 2014.

Nyasa Times, "DPp fears Electoral Fraud, gives Malawi Electoral Body 48 hours Ultimum on Civic Education", 11 de julio de 2013.

Nyasa Times Reporter, "Malawi Voting opens with Numerous Complaints", Nyasa Times, 20 de mayo de 2014.

Olwethu MJamba, Khanyo, "Madonna makes Statement on Malawi President", This is Africa, 27 de mayo de 2014.

Public Affairs Committee, Lilongwe Peace Declaration, Lilongüe, 10 de mayo de 2014. [ < http://peacemaker.un.org/sites/peacemaker.un.org/files/MW_140510_LilongwePeaceDeclaration. pdf $>$, consultado en enero de 2015.]

Reuters, "Citing Fraud, Malawi Leader Halts Vote and Stirs Protest", The New York Times, 24 de mayo de 2014.

SAPA-AFP, "Mutharika wins Malawi's Disputed Presidential Poll", Mail \& Guardian, 31 de mayo de 2014.

Southern African Development Community, SADC Protocol on Gender and Development, 17 de agosto de 2008. [< http://www.wlsa.org. $\mathrm{mz} / \mathrm{wp}$-content/uploads/2014/11/SADC_Protocol_Gender. pdf $>$, consultado en enero de 2015.]

Star Africa, "Malawi pols Extended to Third Day", 22 de mayo de 2014.

The Nation, "MEC broke, Elections under Threat", 17 de mayo de 2013.

Transparency International, Corruption Perceptions Index 2013. [<http://cpi.transparency.org/cpi2013/results/ >, consultado en enero de 2015.]

Ufulu, "Malawi Army and Police assure Malawians of a Secure and Safe Election", The Maravi Post, 20 de mayo de 2014.

United Nations Development Programme, "Malawi Electoral Cycle Support", s.f. [<http://www.mw.undp.org/content/malawi/ en/home/operations/projects/democratic_governance/malawielectoral-cycle-support-/ > , consultado en enero de 2015.]

VARELA, Hilda, "Las elecciones generales de 2004: Sudáfrica y Malawi”, Estudios de Asia y África, vol. 39, núm. 2 (124), mayo-agosto de 2004, pp. 421-444.

Xinhua News Agency, "China congratulates Malawi's New President”, People's Daily Online, 3 de junio de 2014. [ < http:// english.peopledaily.com.cn/n/2014/0603/c90883-8736312. html >, consultado en enero de 2015.] 
